\title{
Properties of Bott manifolds and cohomological rigidity
}

\author{
SUYOUNG CHOI \\ DONG YOUP SUH
}

\begin{abstract}
The cohomological rigidity problem for toric manifolds asks whether the integral cohomology ring of a toric manifold determines the topological type of the manifold. In this paper, we consider the problem with the class of one-twist Bott manifolds to get an affirmative answer to the problem. We also generalize the result to quasitoric manifolds. In doing so, we show that the twist number of a Bott manifold is welldefined and is equal to the cohomological complexity of the cohomology ring of the manifold. We also show that any cohomology Bott manifold is homeomorphic to a Bott manifold. All these results are also generalized to the case with $\mathbb{Z}_{(2)}$-coefficients, where $\mathbb{Z}_{(2)}$ is the localized ring at 2 .
\end{abstract}

$57 \mathrm{~S} 25 ; 22 \mathrm{~F} 30$

\section{Introduction}

A class $\mathcal{M}$ of closed manifolds is said to be cohomologically rigid if any two elements $M, N \in \mathcal{M}$ are homeomorphic whenever their cohomology rings are isomorphic. One of the interesting problems in toric topology is to determine whether the class of toric (or quasitoric) manifolds is cohomologically rigid. A quasitoric manifold is a topological analogue of a toric manifold, which was first introduced by Davis and Januszkiewicz [7] (see also Buchstaber and Panov [2]).

Since the class of toric or quasitoric manifolds is too large to handle, it is reasonable to restrict our attention to a smaller but interesting subclass of manifolds. Namely, we would like to restrict our focus to Bott manifolds or cohomology Bott manifolds.

A (complex) Bott tower $\left\{B_{j} \mid j=0, \ldots, n\right\}$ of height $n$ (or an $n$-stage Bott tower) is a sequence,

$$
B_{n} \stackrel{\pi_{n}}{\longrightarrow} B_{n-1} \stackrel{\pi_{n-1}}{\longrightarrow} \cdots \stackrel{\pi_{2}}{\longrightarrow} B_{1} \stackrel{\pi_{1}}{\longrightarrow} B_{0}=\{\text { a point }\}
$$

of manifolds $B_{j}=P\left(\underline{\mathbb{C}} \oplus \xi_{j-1}\right)$, where $\xi_{j-1}$ is a complex line bundle over $B_{j-1}$ for each $j=1, \ldots, n$. In this case we call $B_{j}$ the $j$ th stage Bott manifold of the Bott tower. A smooth manifold $M$ diffeomorphic to the top stage $B_{n}$ of a Bott tower is 
also called a Bott manifold, and, in this case, $\left\{B_{j} \mid j=0, \ldots n\right\}$ is called a Bott tower structure of $M$.

The Bott towers was first introduced by Bott and Samelson [1] and later named a Bott tower by Grossberg and Karshon [8]. Bott manifolds are known to have algebraic torus actions; hence, they constitute an important family of toric manifolds. A cohomology Bott manifold is a quasitoric manifold whose cohomology ring is isomorphic to that of a Bott manifold.

The question we are interested in here is whether the class of (cohomology) Bott manifolds is cohomologically rigid. So far, there is no counter example to the question, but some positive results. Masuda and Panov considered the problem and showed that any $n$-stage Bott manifold is diffeomorphic to the trivial Bott manifold $\left(\mathbb{C} P^{1}\right)^{n}$ if its cohomology ring is isomorphic to that of $\left(\mathbb{C} P^{1}\right)^{n}$.

The notion of Bott tower was generalized to a generalized Bott tower by the authors and Masuda [4], which is an iterated complex projective space bundles obtained from the projectivization of the sum of line bundles over the total space of the lower stage bundle, and the result of Masuda and Panov [12] was extended to generalized Bott manifolds by the authors and Masuda [5]. Furthermore, any three-stage Bott manifolds and two-stage generalized Bott manifolds are shown to be cohomologically rigid there.

Davis and Januszkiewicz [7] also introduced a real analogue of a quasitoric manifold called a small cover. However, for small covers, the corresponding cohomology rings are with $\mathbb{Z}_{2}$-coefficients. Moreover, we can define a real Bott tower to be iterated $\mathbb{R} P^{1}$ bundles, and a generalized real Bott tower is defined similarly. So one might ask a similar cohomological rigidity question on whether two real Bott manifolds are homeomorphic if their mod 2 cohomology rings are isomorphic. This is shown to be true recently by Kamishima and Masuda [10] (see also the paper by the first author, Masuda and Oum [3]). However, the same question for generalized real Bott manifolds is not true, see Masuda [11].

However, not much is known about the cohomological rigidity of Bott manifolds whose cohomology rings are not isomorphic to that of the products of $\mathbb{C} P^{1}$. In this article we consider a special type of Bott manifolds. The twist number of a Bott tower (1-1) is the number of nontrivial topological fibration $\pi_{i}: B_{i} \rightarrow B_{i-1}$ in the sequence. Since a Bott manifold may have more than one different Bott tower structure, the twist number of a Bott manifold may not be well-defined. However, we show in Theorem 3.2 that the twist number of any Bott tower structure for a Bott manifold $M$ is equal to the cohomological complexity of $M$, which is the number depending only on the cohomology of $M$. See Section 3 for the definition of cohomological complexity of a Bott manifold. In particular, the twist number of a Bott manifold is well-defined. 
We prove in Theorem 5.2 that the class of one-twist Bott manifolds are cohomologically rigid. Moreover, this result is extended to quasitoric manifolds whose $\mathbb{Z}-$ cohomology rings are isomorphic to those of one-twist Bott manifolds in Theorem 5.4. Theorem 5.4 is an immediate consequence of Theorem 5.2 together with Theorem 3.2 and Theorem 4.2.

A BQ-algebra of rank $n$ is defined by Masuda and Panov [12]. In particular, the cohomology ring of any $n$-stage Bott manifold is a BQ-algebra of rank $n$ over $\mathbb{Z}$. Theorem 4.2 says that the converse is also true; namely, any BQ-algebra of rank $n$ over $\mathbb{Z}$ is the cohomology ring of an $n$-stage Bott manifold.

It was proved by the authors and Masuda [5] that the class of three-stage Bott manifolds are cohomologically rigid. An immediate consequence of this result together with Theorem 4.2 is Theorem 4.3, which says that the class of 6-dimensional quasitoric manifolds whose cohomologies are BQ-algebras over $\mathbb{Z}$ is cohomologically rigid.

So far, all the cohomology rings that we studied are cohomologies with $\mathbb{Z}$ coefficients, but by careful observation of the proofs we can see that the same conclusion can be derived with the 2-localized $\mathbb{Z}_{(2)}$-coefficients. This is discussed in Section 6.

Throughout this paper, the integral cohomology ring of $X$ is denote by $H^{*}(X)$ instead of $H^{*}(X: \mathbb{Z})$ for simplicity.

\section{A sum of two line bundles over Bott manifolds}

Let $\left\{B_{j}=P\left(\underline{\mathbb{C}} \oplus \xi_{j-1}\right) \mid 0 \leq j \leq n\right\}$ be a complex Bott tower of height $n$. By the standard results on the cohomology of projectivized bundles, we can see that the cohomology of $B_{j}$ is a free module over $H^{*}\left(B_{j-1}\right)$ on generators 1 and $x_{j}$ of dimensions 0 and 2, respectively. The ring structure of $H^{*}\left(B_{j}\right)$ is determined by a single relation,

$$
x_{j}^{2}=c_{1}\left(\xi_{j-1}\right) x_{j},
$$

where $x_{j}$ is the first Chern class of the line bundle $\gamma_{j}$ that is the pull-back bundle of the tautological line bundle of $P\left(\mathbb{C} \oplus \xi_{j-1}\right)=B_{j}$ via the projection $B_{n} \rightarrow B_{j}$. Since $c_{1}\left(\xi_{j-1}\right) \in H^{2}\left(B_{j-1}\right)$, we can write

$$
f_{j}:=c_{1}\left(\xi_{j-1}\right)=\sum_{i=1}^{j-1} c_{i j} x_{i}
$$

Therefore we have the ring isomorphism

$$
H^{*}\left(B_{n}\right) \cong \mathbb{Z}\left[x_{1}, \ldots, x_{n}\right] / I,
$$


where $I=\left\langle x_{j}\left(x_{j}-f_{j}\right) \mid j=1, \ldots, n\right\rangle$ and $f_{j}=\sum_{i=1}^{j-1} c_{i j} x_{i}$ with $\operatorname{deg} x_{j}=2$ for all $j$. Since complex line bundles are distinguished by their first Chern classes, the Bott manifold $B_{n}$ is determined by the above list of integers $\left(c_{i j} \mid 1 \leq i<j \leq n\right)$.

It is convenient to organize the integers $c_{i j}$ into an $n \times n$ upper triangular matrix:

$$
\Lambda=\left(\begin{array}{cccc}
0 & c_{12} & \cdots & c_{1 n} \\
& 0 & \cdots & \vdots \\
& & \ddots & c_{n-1 n} \\
& & & 0
\end{array}\right) .
$$

We call it the associated matrix of the Bott tower.

One of the basic questions in vector bundle theory is to determine when two bundles with equal characteristic classes are isomorphic. In particular, we would like to know the answer to the following question. Let $\xi$ and $\eta$ be sums of $k$ complex line bundles over a generalized Bott manifold $B$. Are two bundles $\xi$ and $\eta$ isomorphic if their total Chern classes are equal? The answer is 'yes' when $B$ is a generalized Bott tower and $\eta$ is the trivial bundle, see the paper [5] by the authors and Masuda. In this section, we provide two more affirmative answers to the question. They are Proposition 2.4 and Proposition 2.5. We first need the following lemma. We sometimes confuse a Bott tower with its top stage Bott manifold when they are clear from the context.

Lemma 2.1 If $B_{n}$ is a Bott tower with the associated matrix

$$
\Lambda=\left(\begin{array}{cccc}
0 & c_{12} & \cdots & c_{1 n} \\
& 0 & & \vdots \\
& & \ddots & c_{n-1 n} \\
& & & 0
\end{array}\right)
$$

such that $c_{k k+1}=c_{k k+2}=\cdots=c_{k n}=0$, then $B_{n}$ is diffeomorphic to a Bott tower with the associated matrix

$$
\Lambda^{\prime}=\left(\begin{array}{ccccccccc}
0 & c_{12} & \cdots & c_{1 k-1} & c_{1 k+1} & c_{1 k+2} & \cdots & c_{1 n} & c_{1 k} \\
& 0 & \cdots & c_{2 k-1} & c_{2 k+1} & c_{2 k+2} & \cdots & c_{2 n} & c_{2 k} \\
& & \ddots & \vdots & \vdots & \vdots & \vdots & \vdots & \vdots \\
& & & 0 & c_{k-1 k+1} & c_{k-1 k+2} & \cdots & c_{k-1 n} & c_{k-1 k} \\
& & & 0 & c_{k+1 k+2} & \cdots & c_{k+1 n} & 0 \\
& & & & 0 & \vdots & \vdots & \vdots \\
& & & & & & \ddots & c_{n-1 n} & 0 \\
& & & & & & & 0 & 0 \\
& & & & & & & & 0
\end{array}\right) .
$$


Proof Note that this lemma can be seen by the fact that $B_{n}$ and $B_{n}^{\prime}$ are diffeomorphic if two associated matrices are conjugated by a permutation matrix, see the paper [4] by the authors and Masuda, or Masuda and Panov [12]. It is obvious that

$$
\Lambda^{\prime}=P \Lambda P^{-1},
$$

where $P$ is the permutation matrix corresponding to a permutation

$$
(1,2, \ldots, k-1, n, k, k+1, \ldots, n-1) \text {. }
$$

The following lemma is a special case of Lemma 2.1, but since it will be used several times later, we state it as a separate lemma.

Lemma 2.2 Let $B_{n}$ and $B_{n}^{\prime}$ be two $n$-stage Bott towers. If the associated matrices to them are

$$
\left(\begin{array}{ccccc}
0 & * & * & b_{1} & a_{1} \\
& \ddots & * & \vdots & \vdots \\
& & 0 & b_{n-2} & a_{n-2} \\
& & & 0 & 0 \\
& & & & 0
\end{array}\right) \text { and } \quad\left(\begin{array}{ccccc}
0 & * & * & a_{1} & b_{1} \\
& \ddots & * & \vdots & \vdots \\
& & 0 & a_{n-2} & b_{n-2} \\
& & & 0 & 0 \\
& & & & 0
\end{array}\right) \text {, }
$$

respectively, then $B_{n}$ and $B_{n}^{\prime}$ are diffeomorphic.

Consider an $n$-stage Bott tower as in (1-1) and a $k$-stage Bott tower $\left\{B_{j}^{\prime} \mid j=1, \ldots, k\right\}$ for $k<n$. Assume there is a diffeomorphism $f_{k}: B_{k}^{\prime} \rightarrow B_{k}$. Then, a new Bott manifold $B_{k+1}^{\prime}$ can be obtained by the pull-back of the fibration $\pi_{k+1}: B_{k+1} \rightarrow B_{k}$ via the diffeomorphism $f_{k}$. Then, the induced map $f_{k+1}: B_{k+1}^{\prime} \rightarrow B_{k+1}$ is a diffeomorphism. By inductive application of the above construction, we can obtain a new $n$-stage Bott tower $\left\{B_{j}^{\prime} \mid j=1, \ldots, n\right\}$ such that $B_{\ell}$ is diffeomorphic to $B_{\ell}^{\prime}$ for all $\ell=k+1, \ldots, n$. Therefore, $\left\{B_{j}^{\prime} \mid j=1, \ldots, n\right\}$ is another Bott tower structure for the Bott manifold $B_{n} \cong B_{n}^{\prime}$.

Lemma 2.3 Let $\left\{B_{j} \mid j=1, \ldots, n\right\}$ be an $n$-stage Bott tower whose $k$ th stage fibration $\pi_{k}: B_{k} \rightarrow B_{k-1}$ is topologically trivial. Then, there is another Bott tower structure $\left\{B_{j}^{\prime} \mid j=1, \ldots, n\right\}$ for the Bott manifold $B_{n}$, whose associated $n \times n$ matrix has the zero vector for its $k$ th column.

Proof Note that $B_{k}$ is diffeomorphic to $B_{k-1} \times \mathbb{C} P^{1}=P(\underline{\mathbb{C}} \oplus \underline{\mathbb{C}})$, where $\underline{\mathbb{C}}$ is the

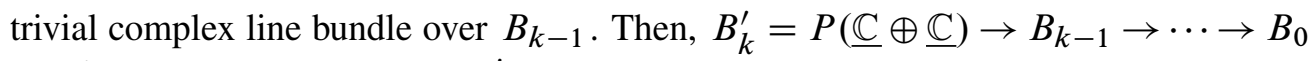
is a $k$-stage Bott tower with $B_{k}^{\prime} \cong B_{k}$. By the above argument, we can find another Bott tower structure $\left\{B_{j}^{\prime} \mid j=1, \ldots, n\right\}$ for the Bott manifold $B_{n}$, whose associated $n \times n$ matrix has the zero vector for its $k$ th column. 
For example, consider the 3-stage Bott tower $B_{3} \rightarrow B_{2} \rightarrow B_{1} \rightarrow B_{0}$ with the associated matrix

$$
\left(\begin{array}{ccc}
0 & 2 a & b \\
0 & 0 & c \\
0 & 0 & 0
\end{array}\right) .
$$

Then, $B_{2} \rightarrow B_{1} \rightarrow B_{0}$ is a 2 -stage Bott tower with the associated matrix

$$
\left(\begin{array}{cc}
0 & 2 a \\
0 & 0
\end{array}\right)
$$

and $B_{2}$ is a Hirzebruch surface. It is well-known that $B_{2} \rightarrow B_{1}$ is topologically a trivial fibration so that $B_{2}$ is diffeomorphic to $B_{2}^{\prime}=\mathbb{C} P^{1} \times \mathbb{C} P^{1}=P(\underline{\mathbb{C}} \oplus \underline{\mathbb{C}})$, where $\underline{\mathbb{C}}$ is the trivial complex line bundle over $\mathbb{C} P^{1}$. Therefore, the Bott manifold $B_{3}$ has another Bott tower structure $B_{3}^{\prime} \rightarrow B_{2}^{\prime} \rightarrow B_{1} \rightarrow B_{0}$ whose associated $3 \times 3$ matrix has the zero second column. More precisely, its associated matrix is

$$
\left(\begin{array}{ccc}
0 & 0 & b+a c \\
0 & 0 & c \\
0 & 0 & 0
\end{array}\right) .
$$

Proposition 2.4 A sum of two line bundles over a Bott manifold is trivial if and only if its total Chern class is trivial.

Proof Let $B_{n}$ be a Bott manifold with the associated matrix

$$
\Lambda=\left(\begin{array}{cccc}
0 & c_{12} & \cdots & c_{1 n} \\
& 0 & & \vdots \\
& & \ddots & c_{n-1 n} \\
& & & 0
\end{array}\right) .
$$

As before, let $x_{j}$ be the first Chern class of the line bundle $\gamma_{j}$, that is, the pull-back bundle of the tautological line bundle of $P\left(\mathbb{C} \oplus \xi_{j-1}\right)=B_{j}$ via the projection $B_{n} \rightarrow B_{j}$. Let $f_{j}=\sum_{i=1}^{j-1} c_{i j} x_{i}$. For an element $\alpha \in H^{2}\left(B_{n}\right)$, let $\gamma^{\alpha}$ be the complex line bundle over $B_{n}$ with $c_{1}\left(\gamma^{\alpha}\right)=\alpha$. Let $\xi=\gamma^{\alpha} \oplus \gamma^{\beta}$ be the sum of two line bundles such that $c(\xi)=1$, and $\alpha=\sum_{j=1}^{n} a_{j} x_{j}$ and $\beta=\sum_{j=1}^{n} b_{j} x_{j}$. Then,

$$
\begin{aligned}
1=c(\xi) & =c\left(\gamma^{\alpha}\right) c\left(\gamma^{\beta}\right) \\
& =(1+\alpha)(1+\beta) \\
& =1+(\alpha+\beta)+\alpha \beta .
\end{aligned}
$$


Therefore, $\alpha+\beta=0$ and $\alpha \beta=0$, which implies $\alpha^{2}=0$ in $H^{*}\left(B_{n}\right)$. On the other hand,

$$
\begin{aligned}
& \alpha^{2}=0 \text { in } H^{*}\left(B_{n}\right), \\
& \Leftrightarrow \sum_{j=1}^{n}\left(a_{j} x_{j}\right)^{2}+\sum_{1 \leq i<j \leq n} 2 a_{j} a_{i} x_{j} x_{i}=\sum_{j=1}^{n} a_{j}^{2}\left(x_{j}^{2}-f_{j} x_{j}\right) \text { as polynomials, }
\end{aligned}
$$

$$
\Leftrightarrow a_{j}^{2} c_{i j}=-2 a_{j} a_{i} \text { for all } i<j \text {. }
$$

Thus, $\xi=\gamma^{\alpha} \oplus \gamma^{-\alpha}$ with $\alpha=\sum_{j=1}^{n} a_{j} x_{j} \in H^{2}\left(B_{n}\right)$ and $a_{j}^{2} c_{i j}=-2 a_{j} a_{i}$ for all $1 \leq i<j \leq n$.

Now, we prove the proposition by induction on $n$. If $n=2$, then the dimension of $\xi$ is equal to the dimension of $B_{n}$, so we are in the stable range. Hence, the total Chern class classifies the complex vector bundle, so the proposition is true for $n=2$. Assume the lemma is true for $B_{n-1}$. We now prove the lemma for $B_{n}$. These are three cases to consider

Case $1 a_{n}=0$.

In this case, $\xi=\pi_{n}^{*}(\eta)$, where $\eta=\gamma^{\alpha} \oplus \gamma^{-\alpha}$ over $B_{n-1}$. By the assumption, $c(\eta)=1$, and by the induction hypothesis, $\eta$ is trivial. So is $\xi$.

Case $2 a_{n} \neq 0$ and $a_{k}=0$ for some $k<n$.

We may assume that $a_{i} \neq 0$ for all $i>k$. By (2-3), $a_{j}^{2} c_{i j}=-2 a_{j} a_{i}$ for all $i<j$. Hence, $a_{k+\ell}^{2} c_{k k+\ell}=-2 a_{k+\ell} a_{k}$ for all $0<\ell \leq n-k$. Since $a_{k+\ell} \neq 0$ and $a_{k}=0, c_{k k+\ell}=0$ for all $\ell$. Thus, $c_{k k+1}=c_{k k+2}=\cdots=c_{k n}=0$. Hence, $B_{n}$ is diffeomorphic to a Bott manifold $B_{n}^{\prime}$ with $\Lambda^{\prime}$ in Lemma 2.1 as the associated matrix.

Let $\left\{x_{1}, \ldots, x_{n}\right\}$ and $\left\{y_{1}, \ldots, y_{n}\right\}$ be ordered generator sets of $H^{*}\left(B_{n}\right)$ and $H^{*}\left(B_{n}^{\prime}\right)$ respectively, as in (2-1). Let $\rho: B_{n}^{\prime} \rightarrow B_{n}$ be the diffeomorphism as indicated in the proof of Lemma 2.1. Then, we can see that

$$
\begin{aligned}
\rho^{*}\left(x_{1}\right) & =y_{1} \\
\vdots & \\
\rho^{*}\left(x_{k-1}\right) & =y_{k-1} \\
\rho^{*}\left(x_{k}\right) & =y_{n} \\
\rho^{*}\left(x_{k+1}\right) & =y_{k} \\
\vdots & \\
\rho^{*}\left(x_{n}\right) & =y_{n-1} .
\end{aligned}
$$


Therefore, $\rho^{*}(\alpha)=a_{1} y_{1}+\cdots+a_{k-1} y_{k-1}+a_{k+1} y_{k}+\cdots+a_{n} y_{n-1}+a_{k} y_{n}$. Since $c\left(\gamma^{\alpha} \oplus \gamma^{-\alpha}\right)=0$ in $H^{*}\left(B_{n}\right), c\left(\rho^{*}\left(\gamma^{\alpha} \oplus \gamma^{-\alpha}\right)\right)=0$ in $H^{*}\left(B_{n}^{\prime}\right)$. Since $a_{k}=0$ from the assumption, we are in Case 1 for $B_{n}^{\prime}$. Therefore, $\rho^{*}\left(\gamma^{\alpha} \oplus \gamma^{-\alpha}\right)$ is trivial on $B_{n}^{\prime}$, and so is $\gamma^{\alpha} \oplus \gamma^{-\alpha}$ on $B_{n}$.

Case $3 a_{j} \neq 0$ for all $j$.

By (2-3), $a_{j}^{2} c_{i j}=-2 a_{j} a_{i}$ for all $i<j$; hence, $c_{i j} \neq 0$ for all $i, j$. Note that $B_{2}$ is a Hirzebruch surface, and the diffeomorphism type of a Hirzebruch surface $B_{2}$ is determined by the parity of $c_{12}$. Hence, if $c_{12}$ is even, by the argument of Lemma 2.3, the Bott manifold $B_{n}$ has another Bott tower structure $\left\{B_{j}^{\prime} \mid j=1, \ldots, n\right\}$ with the associated matrix

$$
\Lambda^{\prime}=\left(\begin{array}{cccc}
0 & c_{12}^{\prime} & \cdots & c_{1 n}^{\prime} \\
& 0 & & \vdots \\
& & \ddots & c_{n-1 n}^{\prime} \\
& & & 0
\end{array}\right)
$$

such that $c_{12}^{\prime}=0$. In particular, there is a diffeomorphism $f_{n}: B_{n}^{\prime} \rightarrow B_{n}$. Since the total Chern class of $\xi$ is trivial, clearly the total Chern class of the pull-back bundle $f_{n}^{*}(\xi)$ is trivial, too. Therefore, if we prove that the pull-back bundle $f_{n}^{*}(\xi)$ over $B_{n}^{\prime}$ is trivial, then $\xi$ is also trivial. Therefore, for simplicity we may assume that $c_{12}=0$.

But then by (2-3), either $a_{1}$ or $a_{2}$ is zero, which contradicts to the assumption of Case 3. Therefore, $c_{12}$ is odd. By the same argument used above, we may assume that $c_{12}=1$.

Since $c_{i j} \neq 0$ and $a_{j} \neq 0$ for all $j$ and $i$, by (2-3), $c_{i j} a_{j}=-2 a_{i}$ for all $i<j$. Hence, $a_{2}=-2 a_{1}$. Moreover, since $c_{13} a_{3}=-2 a_{1}$, we have $c_{23} a_{3}=-2 a_{2}=4 a_{1}$; hence, $c_{23}=-2 c_{13}$. We claim that $B_{3}$ with

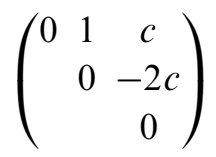

is diffeomorphic to $B_{3}^{\prime}$ with

$$
\left(\begin{array}{lll}
0 & 1 & c \\
& 0 & 0 \\
& & 0
\end{array}\right) .
$$

Thus, we may assume that $B_{n}$ has $c_{23}=0$. Then, by (2-3), $a_{2}$ or $a_{3}$ must be zero. Therefore, we are in Case 2, and the proposition is proved. 
The claim remains to be proven.

$$
\begin{aligned}
B_{3} & =P\left(\mathbb{C} \oplus\left(\gamma_{1}^{c} \otimes \gamma_{2}^{-2 c}\right)\right) \\
& \cong P\left(\left(\mathbb{C} \oplus\left(\gamma_{1}^{c} \otimes \gamma_{2}^{-2 c}\right)\right) \otimes \gamma_{2}^{c}\right) \\
& \cong P\left(\gamma_{2}^{c} \oplus\left(\gamma_{1}^{c} \otimes \gamma_{2}^{-c}\right)\right) .
\end{aligned}
$$

The total Chern class of $\gamma_{2}^{c} \oplus\left(\gamma_{1}^{c} \otimes \gamma_{2}^{-c}\right)$ is

$$
c\left(\gamma_{2}^{c} \oplus\left(\gamma_{1}^{c} \otimes \gamma_{2}^{-c}\right)\right)=\left(1+c x_{2}\right)\left(1+c x_{1}-c x_{2}\right)=1+c x_{1}
$$

since $x_{2}^{2}=x_{1} x_{2}$ in $H^{4}\left(B_{2}\right)$.

On the other hand, $c\left(\mathbb{C} \oplus \gamma_{1}^{c}\right)=1+c x_{1}$. Therefore, $\gamma_{2}^{c} \oplus\left(\gamma_{1}^{c} \otimes \gamma_{2}^{-c}\right) \cong \mathbb{C} \oplus \gamma_{1}^{c}$ as bundles over $B_{2}$. Thus, $B_{3} \cong P\left(\mathbb{C} \oplus \gamma_{1}^{c}\right)=B_{3}^{\prime}$, which has the associated matrix

$$
\left(\begin{array}{ccc}
0 & 1 & c \\
& 0 & 0 \\
& & \\
& & 0
\end{array}\right) \text {. }
$$

This completes the proof.

Now let $B_{n-1} \cong\left(\mathbb{C} P^{1}\right)^{n-1}$, and, for $\alpha \in H^{2}\left(B_{n-1}\right)$, let $\gamma^{\alpha}$ be the complex line bundle over $B_{n-1}$ with $c_{1}\left(\gamma^{\alpha}\right)=\alpha$ as before.

Proposition 2.5 Let $\xi_{1}=\gamma^{\alpha_{1}} \oplus \gamma^{\alpha_{2}}$ and $\xi_{2}=\gamma^{\beta_{1}} \oplus \gamma^{\beta_{2}}$ be the sums of two line bundles over $B_{n-1} \cong\left(\mathbb{C} P^{1}\right)^{n-1}$ such that $c_{1}\left(\xi_{1}\right)=c_{1}\left(\xi_{2}\right)$ and $c_{2}\left(\xi_{1}\right)=c_{2}\left(\xi_{2}\right)=0$. Then, $\xi_{1}$ and $\xi_{2}$ are isomorphic.

Proof Let $H^{*}\left(B_{n-1}\right) \cong \mathbb{Z}\left[x_{1}, \ldots, x_{n-1}\right] /\left\langle x_{j}^{2} \mid j=1, \ldots, n-1\right\rangle$, and let $\alpha_{k}, \beta_{k}$ be elements of $H^{2}\left(B_{n-1}\right)$ for $k=1,2$. From the assumption, we have $\alpha_{1}+\alpha_{2}=\beta_{1}+\beta_{2}$ and $\alpha_{1} \alpha_{2}=\beta_{1} \beta_{2}=0$.

In general, for two elements, $u=\sum_{i=1}^{n-1} u_{i} x_{i}$ and $v=\sum_{i=1}^{n-1} v_{i} x_{i}$ of $H^{2}\left(B_{n-1}\right)$, the identity $u v=0$ holds if and only if $u_{j} v_{i}+u_{i} v_{j}=0$ for any $j \neq i$. From this, we can see easily that if $u v=0$, one of the following three possibilities follows.

(1) If at least three coefficients in $u$ are non-zero, then $v=0$.

(2) If exactly two coefficients in $u$, say $u_{i}$ and $u_{j}$, are non-zero, then $v$ is either 0 or has exactly two non-zero coefficients with $v_{j} v_{i} \neq 0$ and $u_{j} v_{i}+u_{i} v_{j}=0$.

(3) If only one coefficient in $u$, say $u_{j}$, is non-zero, then $v$ is either 0 or has exactly one non-zero coefficient with $v_{j} \neq 0$. 
Suppose $\alpha_{1}$ has at least three non-zero coefficients. Then, (1) implies that $\alpha_{2}=0$ and $\alpha_{1}=\beta_{1}+\beta_{2}$. If both $\beta_{1}$ and $\beta_{2}$ are non-zero, then they should have at most two non-zero coefficients at the same places by (1), (2), and (3), which contradicts the assumption that $\alpha_{1}$ has at least three nonzero coefficients because $\alpha_{1}+\alpha_{2}=\beta_{1}+\beta_{2}$. Therefore, either $\beta_{1}=0$ or $\beta_{2}=0$, and two bundles, $\xi_{1}$ and $\xi_{2}$, are isomorphic.

Suppose that $\alpha_{1}$ has exactly two non-zero coefficients. Then, (1) and (2) imply that $\alpha_{2}, \beta_{1}$, and $\beta_{2}$ are either zero or have exactly two non-zero coefficients at the same places as $\alpha_{1}$. This means that the bundles $\xi_{1}$ and $\xi_{2}$ are pullbacks of bundles over $\left(\mathbb{C} P^{1}\right)^{2}$. Hence, those bundles are in a stable range, and hence, they are classified by their Chern classes. Thus, $\xi_{1}$ and $\xi_{2}$ are isomorphic.

The case when $\alpha_{1}$ has only one non-zero coefficient can be similarly proved.

\section{Twist number and cohomological complexity}

The twist number of a Bott tower $\left\{B_{j} \mid j=0, \ldots, n\right\}$ is the number of nontrivial topological fibrations $B_{j} \rightarrow B_{j-1}$ in the sequence. However there may be several Bott tower structures for a Bott manifold, so the twist number may not be well-defined for Bott manifolds. In this section, we show that the twist number of a Bott manifold is well-defined; namely, we show that the twist numbers of any Bott tower structure of a Bott manifold is constant. A Bott tower with the twist number equal to $t$ is simply called a $t$-twist Bott tower.

Lemma 3.1 If a Bott manifold has a $t$-twist Bott tower structure, then it has another Bott tower structure whose last $t$ stages are nontrivial and all other stages are trivial.

Proof Let $\left\{B_{j} \mid j=1, \ldots, n\right\}$ be a Bott tower structure of a Bott manifold $B_{n}$ with the twist number equal to $t$, and let $\Lambda=\left(c_{i j}\right)_{i, j=1, \ldots, n}$ be the associated $n \times n$ matrix. If the $k$ th stage $\pi_{k}: B_{k} \rightarrow B_{k-1}$ is topologically a trivial fibration, then by choosing a different Bott tower structure of $B_{n}$, if necessary, we may assume that the $k$ th column of $\Lambda$ is zero. Now consider the $k \times k$ submatrix $\Lambda_{k}=\left(c_{i j}\right)_{i, j=1, \ldots, k}$ of $\Lambda$, which is the associated matrix of the Bott subtower $\left\{B_{i} \mid i=1, \ldots, k\right\}$. Then, the $k$ th column of $\Lambda_{k}$ is also zero. By Lemma 2.2, there is another Bott tower structure $\left\{B_{i}^{\prime} \mid i=1, \ldots, k\right\}$ for the Bott manifold $B_{k}$, whose associated matrix has the zero $(k-1)$ st column. In particular the $(k-1)$ st fibration is trivial. By Lemma 2.3, we can find a Bott tower structure $\left\{B_{j}^{\prime} \mid j=1, \ldots, n\right\}$ for the Bott manifold $B_{n}$, whose $(k-1)$ st stage is topologically a trivial fibration. In other words, by choosing a different Bott tower structure of a Bott manifold, we can push the non-first trivial stage of the fibration down by one stage. By successive application of the above argument, we can 
find an appropriate Bott tower structure for the given Bott manifold $B_{n}$ whose top $t$ stages are nontrivial but the all other lower stage fibrations are trivial. This proves the lemma.

Let $M$ be a Bott manifold, and let $\left\{B_{j} \mid j=1, \ldots, n\right\}$ be a Bott tower structure on $M$; hence, $M \cong B_{n}$. Then, the cohomology ring of $M$ is isomorphic to

$$
H^{*}(M) \cong \mathbb{Z}\left[x_{1}, \ldots, x_{n}\right] / I,
$$

where $I=\left\langle x_{j}\left(x_{j}-f_{j}\right) \mid j=1, \ldots, n\right\rangle$ and $f_{j}=\sum_{i=1}^{j-1} c_{i j} x_{i}$ with $\operatorname{deg} x_{j}=2$. Here, the numbers $c_{i j}$ can be determined by the Bott tower structure $\left\{B_{j} \mid j=1, \ldots, n\right\}$ of $M$. Indeed, $c_{i j}$ 's are the entries of the matrix (2-2). Note that the number of nonzero $f_{j}$ 's depends not only on the choice of Bott tower structure $\left\{B_{j} \mid j=1, \ldots, n\right\}$ on $M$, but also the generators $x_{j}$ 's of the cohomology $H^{*}(M)$ of $M$. We now define the cohomological complexity of $M$ to be the minimum number of nonzero $f_{j}$ 's among all possible choices of both Bott tower structures of $M$ and the generators of the cohomology ring of $M$. Then, from the definition, it is clear that the cohomological complexity of $M$ depends only on the topology of $M$, and, hence, it is independent of the choice of Bott tower structure on $M$.

In the following theorem, we show that the twist number of any Bott tower structure of a Bott manifold $M$ is equal to the cohomological complexity of $M$. Since the cohomological complexity is independent of the choice of Bott tower structure on $M$ the twist numbers of any two Bott tower structures of a Bott manifolds are identical. Thus, the twist number of a Bott manifold is well-defined.

Theorem 3.2 Let $M$ be a Bott manifold. Then, the twist number of any Bott tower structure of $M$ is equal to the cohomological complexity of $M$.

\section{Proof Let}

$$
B_{n} \rightarrow B_{n-1} \rightarrow \cdots \rightarrow B_{1} \rightarrow B_{0}=\{\text { a point }\}
$$

be a Bott tower structure of $M$, whose twist number is equal to $t$. By Lemma 3.1, we may assume that

$$
B_{n-t} \rightarrow B_{n-t-1} \rightarrow \cdots \rightarrow B_{1}
$$

is a trivial Bott tower. Therefore, $B_{\ell}=\left(\mathbb{C} P^{1}\right)^{\ell}$ for $\ell=1, \ldots, n-t$.

Let $s$ be the cohomological complexity of $M$. Then, it is clear that $t \geq s$ in general. Suppose $t>s$. Since the twist number of $M$ is $t$, we have

$$
H^{*}\left(B_{n}\right)=\mathbb{Z}\left[x_{1}, \ldots, x_{n}\right] /\left\langle x_{j}\left(x_{j}-f_{j}\right) \mid j=1, \ldots, n\right\rangle,
$$


where

$$
f_{j}= \begin{cases}0 & \text { for } 1 \leq j \leq n-t \\ \sum_{i=1}^{j-1} c_{i j} x_{i} & \text { for } n-t<j \leq n\end{cases}
$$

Since the cohomological complexity of $B_{n}$ is $s$, there is an isomorphism

$$
\psi: H^{*}\left(B_{n}\right) \rightarrow \mathbb{Z}\left[y_{1}, \ldots, y_{n}\right] /\left\langle y_{j}\left(y_{j}-g_{j}\right) \mid j=1, \ldots, n\right\rangle,
$$

where

$$
g_{j}= \begin{cases}0 & \text { for } 1 \leq j \leq n-s, \\ \sum_{i=1}^{j-1} d_{i j} y_{i} & \text { for } n-s<j \leq n\end{cases}
$$

We claim that there exists $m(n-t<m \leq n)$ such that $f_{m} \equiv 0 \bmod 2$ and $f_{m}^{2}=$ $0 \in H^{*}\left(B_{m-1}\right)$. If the claim is true, then we can write as $f_{m}+2 w=0$ for some $w \in H^{2}\left(B_{m-1}\right)$. Therefore,

$$
\begin{aligned}
c\left(\gamma^{w} \oplus \gamma^{f_{m}+w}\right) & =(1+w)\left(1+f_{m}+w\right)=1+\left(f_{m}+2 w\right)-\frac{f_{m}^{2}}{4} \\
& =1 .
\end{aligned}
$$

Thus, by Proposition 2.4, $\gamma^{w} \oplus \gamma^{f_{m}+w}$ is a trivial bundle over $B_{m-1}$. Hence, $P(\mathbb{C} \oplus$ $\left.\gamma^{f_{m}}\right)=P\left(\gamma^{w} \oplus \gamma^{f_{m}+w}\right)=B_{m-1} \times \mathbb{C} P^{1}$. So we can reduce the twist number of $B_{n}$ to $t-1$, which is a contradiction.

We now prove the claim. Since $\psi$ is an isomorphism, we can write

$$
y_{i}=\sum_{j=1}^{n} b_{i j} \psi\left(x_{j}\right) .
$$

Let $B=\left(b_{i j}\right)$ be the coefficient matrix. Note that $\operatorname{det}(B)= \pm 1$.

Since $\psi^{-1}\left(y_{k}^{2}\right)=0$ in $H^{*}\left(B_{n}\right)$ for $1 \leq k \leq n-s$, we have

$$
\begin{aligned}
\psi^{-1}\left(y_{k}^{2}\right) & =\left(\psi^{-1}\left(y_{k}\right)\right)^{2}=\left(\sum_{j=1}^{n} b_{k j} x_{j}\right)^{2} \\
& =\sum_{j=1}^{n}\left(b_{k j}\right)^{2} x_{j}^{2}+\sum_{1 \leq i<j \leq n} 2 b_{k j} b_{k i} x_{i} x_{j} \\
& =\sum_{j=1}^{n}\left(b_{k j}\right)^{2}\left(x_{j}^{2}-f_{j} x_{j}\right), \text { which represents zero in } H^{*}\left(B_{n}\right) \\
& =\sum_{j=1}^{n}\left(b_{k j}\right)^{2}\left(x_{j}^{2}-\sum_{i=1} c_{i j} x_{i} x_{j}\right) .
\end{aligned}
$$


By comparing the coefficients of (3-1) and (3-2), we have

$$
\sum_{i=1}^{j-1} 2 b_{k j} b_{k i} x_{i}=-\left(b_{k j}\right)^{2} f_{j}
$$

for $1 \leq k \leq n-s$ and $1 \leq j \leq n$. This implies

$$
2 b_{k j} b_{k i}=-\left(b_{k j}\right)^{2} c_{i j},
$$

where $1 \leq k \leq n-s$ and $1 \leq i<j$.

Suppose that all $b_{k j}$ are even for $n-t+1 \leq j \leq n$ and $1 \leq k \leq n-s$. Since $t+n-s>n$, det $B$ must be even because, in general, if $A=\left(\begin{array}{cc}C & D \\ E & F\end{array}\right)$ is an $n \times n$ matrix and if $D$ is a $k \times \ell$ matrix all of whose entries are even with $k+\ell>n$, then $\operatorname{det} B$ is even. This is a contradiction. Thus, there is an odd number $b_{\ell m}$ for some $1 \leq \ell \leq n-s$ and $n-t+1 \leq m \leq n$.

Suppose $f_{m}$ is not congruent to 0 modulo 2; that is, there exists an odd number $c_{h m}$ for some $1 \leq h \leq m-1$. Then, from (3-4), $2 b_{k m} b_{k h}=-\left(b_{k m}\right)^{2} c_{h m}$ for $1 \leq k \leq n-s$. It implies that $b_{k m} \equiv 0(\bmod 2)$ for all $1 \leq k \leq n-s$, which contradicts the notion that $b_{\ell m}$ is odd. Thus, $f_{m} \equiv 0(\bmod 2)$.

On the other hand, from (3-3), $\sum_{i=1}^{m-1} 2 b_{k m} b_{k i} x_{i}=-\left(b_{k m}\right)^{2} f_{m}$ with $k=\ell, \frac{f_{m}}{2}=$ $-\sum_{j=1}^{m-1} \frac{b_{\ell j}}{b_{\ell m}} x_{j}$. Thus we have

$$
\begin{aligned}
\left(\frac{f_{m}}{2}\right)^{2} & =\left(-\sum_{j=1}^{m-1} \frac{b_{\ell j}}{b_{\ell m}} x_{j}\right)^{2} \\
& =\sum_{j=1}^{m-1}\left(\left(\frac{b_{\ell j}}{b_{\ell m}}\right)^{2} x_{j}^{2}+2 \sum_{h=1}^{j-1} \frac{b_{\ell j} b_{\ell h}}{\left(b_{\ell m}\right)^{2}} x_{j} x_{h}\right) \\
& =\frac{\sum_{j=1}^{m-1}\left(b_{\ell j}\right)^{2}\left(x_{j}^{2}-f_{j} x_{j}\right)}{\left(b_{\ell m}\right)^{2}} \\
& =0 \in H^{*}\left(B_{m-1}\right) .
\end{aligned}
$$

This proves the claim.

From the proof of Theorem 3.2, the following corollary follows immediately.

Corollary 3.3 The twist number of a Bott manifold $M$ is well-defined; that is, any two Bott tower structures of $M$ have the same twist number. 


\section{BQ-algebras and Bott manifolds}

Recall that a $2 n$-dimensional manifold $M$ is a quasitoric manifold over a simple (combinatorial) polytope $P$ if there is a locally standard $n$-torus $T^{n}$ action on $M$ and a surjective map $\pi: M \rightarrow P$ whose fibers are the $T^{n}$-orbits. For a $2 n$-dimensional quasitoric manifold $M$ over a simple polytope $P$, there corresponds a characteristic map $\chi: \mathcal{F} \rightarrow \mathbb{Z}^{n}$ that is well-defined up to the sign where $\mathcal{F}$ is the set of all facets of $P$. A characteristic map should satisfy the following two conditions:

- $\chi(F)$ is a primitive vector for any $F \in \mathcal{F}$, and

- if $n$ facets $F_{1} \ldots, F_{n}$ intersect at vertex $v$ of $P$, then $\left\{\chi\left(F_{1}\right), \ldots, \chi\left(F_{n}\right)\right\}$ forms a basis of $\mathbb{Z}^{n}$.

Conversely, for simple polytope $P$ and a map $\chi: \mathcal{F} \rightarrow \mathbb{Z}^{n}$ satisfying the above two conditions, there exists a unique quasitoric manifold up to the equivalence whose characteristic map is $\chi$.

Two quasitoric manifolds $\pi_{M}: M \rightarrow P$ and $\pi_{N}: N \rightarrow P$ over $P$ are equivalent if there is a weak $T^{n}$-equivariant homeomorphism $\phi: M \rightarrow N$ (that is, there exists an automorphism $\rho$ on $T^{n}$ such that $\left.\phi(t x)=\rho(t) \phi(x)\right)$ such that $\pi_{N} \circ \phi=\pi_{M}$.

Let $P$ be an $n$-dimensional simple polytope with $m$ facets, and let $M$ be a quasitoric manifold over $P$. Then, we can find a characteristic map $\chi$ for $M$ such that $\chi\left(F_{1}\right)=$ $(1,0, \ldots, 0), \ldots, \chi\left(F_{n}\right)=(0, \ldots, 0,1)$, where $F_{1}, \ldots, F_{n}$ are the facets meeting at one particular vertex $p \in P$. Then, we can define an $(m-n) \times n$ matrix $A$ whose row vectors are $\chi\left(F_{n+1}\right), \ldots, \chi\left(F_{m}\right)$. This matrix $A$ is called a characteristic matrix of $M$. For details about quasitoric manifolds, we refer the reader to Davis and Januszkiewicz [7]. We note that the Bott manifold $B_{n}$ associated with the matrix $\Lambda$ in (2-2) admits the canonical nice $T^{n}$-action with which $B_{n}$ becomes a quasitoric manifold. The characteristic matrix of $B_{n}$ is then equal to $\Lambda-I_{n}$, where $I_{n}$ is the identity matrix of size $n$ (see Masuda and Panov [12] for details).

In this section, we will consider quasitoric manifolds whose cohomology rings resemble those of Bott manifolds. For this, we need the following definition.

Definition 4.1 A graded algebra $S$ over $\mathbb{Z}$ generated by $x_{1}, \ldots, x_{n}$ of degree 2 is called a Bott quadratic algebra (BQ-algebra) over $\mathbb{Z}$ of rank $n$ if

(1) $x_{k}^{2}=\sum_{i<k} c_{i k} x_{i} x_{k}$ where $c_{i k} \in \mathbb{Z}$ for $1 \leq k \leq n$, (in particular, $x_{1}^{2}=0$ ) and (2) $\prod_{i=1}^{n} x_{i} \neq 0$.

A BQ-algebra over $\mathbb{Z}_{2}$ is defined similarly. 
Originally, a BQ-algebra over $\mathbb{Z}_{2}$ is defined in [12], and we extend their definition here for our purpose. The cohomology ring of a Bott manifold is a BQ-algebra over $\mathbb{Z}$. So one might ask whether the converse is true; that is, if the cohomology ring of a quasitoric manifold is a BQ-algebra over $\mathbb{Z}$, then is the quasitoric manifold homeomorphic to a Bott tower? A stronger affirmative answer to the question is given in the following theorem.

Theorem 4.2 Let $M$ be a $2 n$-dimensional quasitoric manifold over a simple polytope $P$, and let $A$ be a characteristic matrix of $M$. Then, the following are equivalent.

(1) $M$ is equivalent to an $n$-stage Bott manifold.

(2) $H^{*}(M)$ is a $B Q$-algebra of rank $n$ over $\mathbb{Z}$.

(3) $P$ is combinatorially equivalent to the cube $I^{n}$, and $A$ is conjugate to an upper triangular matrix by a permutation matrix.

Proof $(1) \Rightarrow(2)$ Clear.

(3) $\Leftrightarrow$ (1) follows from [12, Proposition 3.2].

(2) $\Rightarrow$ (3) If $H^{*}(M)$ is a BQ-algebra of rank $n$ over $\mathbb{Z}$, then $H^{*}\left(M: \mathbb{Z}_{2}\right)$ is a BQ-algebra of rank $n$ over $\mathbb{Z}_{2}$. According to [12, Theorem 5.5] (or a result of the authors and Panov [6, Theorem 1.6]) $P$ is combinatorially equivalent to the cube $I^{n}$. Therefore, $A$ is an $n \times n$ matrix. We may assume that

$$
-A=\left(\begin{array}{cccc}
1 & a_{12} & \cdots & a_{1 n} \\
a_{21} & 1 & \cdots & a_{2 n} \\
\vdots & \vdots & \ddots & \vdots \\
a_{n 1} & a_{n 2} & \cdots & 1
\end{array}\right) .
$$

We note that the conditions of a characteristic map implies that all principal minors are \pm 1 , and, by general facts on the cohomology of quasitoric manifolds, we have an isomorphism

$$
H^{*}(M) \cong \mathbb{Z}\left[y_{1}, \ldots, y_{n}\right] /\left\langle g_{j} \mid j=1, \ldots, n\right\rangle,
$$

where $g_{j}=y_{j} \sum_{i=1}^{n} a_{i j} y_{i}$ and $a_{j j}=1$ for all $i=1, \ldots, n$. Since $H^{*}(M)$ is a BQ-algebra over $\mathbb{Z}$, there is a $\mathbb{Z}$-algebra isomorphism

$$
\phi: H^{*}(M) \rightarrow \mathbb{Z}\left[x_{1}, \ldots, x_{n}\right] /\left\langle x_{j}\left(x_{j}-f_{j}\right) \mid j=1, \ldots, n\right\rangle,
$$

where $f_{j}=\sum_{i=1}^{j-1} c_{i j} x_{i}$. Therefore,

$$
x_{i}=\sum_{j=1}^{n} b_{i j} \phi\left(y_{j}\right)
$$


with det $B= \pm 1$, where $B$ is the $n \times n$ matrix $\left(b_{i j}\right)$. Since all principal $2 \times 2$ minors of $A$ are \pm 1 by the conditions of characteristic map, we have $1-a_{i j} a_{j i}= \pm 1$ for all $i \neq j$.

We first claim that $a_{i j} a_{j i}=0$ for all $i \neq j$. Assume otherwise. Then $a_{t s} a_{s t}=2$ for some $s$ and $t$. Since $\phi^{-1}\left(x_{1}^{2}\right)=\left(\sum_{j=1}^{n} b_{1 j} y_{j}\right)^{2}=0$ in $H^{*}(M)$, we have

$$
\left(\sum_{j=1}^{n} b_{1 j} y_{j}\right)^{2}=\sum_{j=1}^{n} b_{1 j}^{2}\left(y_{j} \sum_{i=1}^{n} a_{i j} y_{i}\right)
$$

Compare the coefficients of $y_{s} y_{t}$-terms on both sides of Equation (4-1) to get

$$
2 b_{1 s} b_{1 t}=b_{1 s}^{2} a_{t s}+b_{1 t}^{2} a_{s t}
$$

Since $a_{s t} a_{t s}=2$ we have $\left(a_{s t}, a_{t s}\right)= \pm(1,2)$ or $\pm(2,1)$. Therefore, Equation (4-2) is equivalent to either $\left(b_{1 s} \pm b_{1 t}\right)^{2}+b_{1 t}^{2}=0$ or $\left(b_{1 s} \pm b_{1 t}\right)^{2}+b_{1 s}^{2}=0$. The only real solutions for Equation (4-2) is $b_{1 s}=b_{1 t}=0$. Hence, $\phi^{-1}\left(x_{1}\right)=\sum_{j \neq s, t} b_{1 j} y_{j}$.

We now consider the second relation $x_{2}\left(x_{2}-f_{2}\right)$ of the BQ-algebra. Here, $f_{2}=c_{12} x_{1}$. Then, $\phi^{-1}\left(f_{2}\right)=\phi^{-1}\left(c_{12} x_{1}\right)=c_{12} \phi^{-1}\left(x_{1}\right)$ has no $y_{s}$ and $y_{t}$-terms. Note that

$$
\begin{aligned}
\phi^{-1}\left(x_{2}\left(x_{2}-f_{2}\right)\right) & =\phi^{-1}\left(x_{2}\right)^{2}-\phi^{-1}\left(x_{2}\right) \phi^{-1}\left(f_{2}\right) \\
& =\left(\sum_{j=1}^{n} b_{2 j} y_{j}\right)^{2}-\left(\sum_{j=1}^{n} b_{2 j} y_{j}\right) c_{12}\left(\sum_{j \neq s, t} b_{1 j} y_{j}\right) \\
& =0 \in H^{*}(M) .
\end{aligned}
$$

Therefore, we have the following equation.

$$
\left(\sum_{j=1}^{n} b_{2 j} y_{j}\right)^{2}-\left(\sum_{j=1}^{n} b_{2 j} y_{j}\right) c_{12}\left(\sum_{j \neq s, t} b_{1 j} y_{j}\right)=\sum_{j=1}^{n} \alpha_{j} g_{j}
$$

for some $\alpha_{j} \in \mathbb{Z}$ with $j=1, \ldots, n$. Since the second term of the left hand side of Equation (4-3) has no $y_{s} y_{t}$-term, no $y_{s}^{2}$-term, and no $y_{t}^{2}$-term, by comparing the coefficients of $y_{s}^{2}$ and $y_{t}^{2}$, we can see that $\alpha_{s}=b_{2 s}^{2}$ and $\alpha_{t}=b_{2 t}^{2}$. Hence, by comparing the coefficients of $y_{s} y_{t}$ of Equation (4-3) we get

$$
2 b_{2 s} b_{2 t}=a_{t s} \alpha_{s}+a_{s t} \alpha_{t}=a_{t s} b_{2 s}^{2}+a_{t s} b_{2 t}^{2},
$$

which is of the same form as in Equation (4-2). Hence, $b_{2 s}=b_{2 t}=0$. Note that $\phi^{-1}\left(f_{3}\right)$ also has no $y_{s}$ and $y_{t}$-terms. Thus, by the same argument used above, we can see that $b_{3 s}=b_{3 t}=0$. Continue the similar argument for $x_{i}\left(x_{i}-f_{i}\right)$ to get

$$
b_{i s}=b_{i t}=0 \quad \text { for all } i=1, \ldots, n .
$$


This implies that the $s$ th and $t$ th rows of the matrix $B$ are zero, which implies $\operatorname{det} B=0$. This is a contradiction. Therefore, the claim that $a_{i j} a_{j i}=0$ for all $i \neq j$ is proved.

We now claim that all principal minors of $A$ are 1 , by induction on the rank of the minors. By the previous claim, any principal minor of rank 2 is 1 . Assume the claim is true for all principal minors of rank $<k$ with $k \geq 3$. Suppose a negative principal minor $\Xi$ of rank $k$ exists. Since all proper minors of $\Xi$ is 1 and $\Xi=-1$, by Masuda and Panov [12, Lemma 3.3], we have

$$
-1=\Xi=\operatorname{det}\left(\begin{array}{ccccc}
1 & h_{j_{1}} & 0 & \ldots & 0 \\
0 & 1 & h_{j_{2}} & \ldots & 0 \\
\vdots & & \ddots & & \vdots \\
0 & \ldots & \ldots & 1 & h_{j_{k-1}} \\
h_{j_{k}} & 0 & & \ldots & 1
\end{array}\right) \text {, }
$$

where $h_{i_{i}} \neq 0$ for all $i=1, \ldots, k$. Consider Equation (4-1) again, but now compare the coefficients of $y_{j_{i}} y_{j_{i+1}}$, where $y_{j_{k+1}}=y_{j_{1}}$ for convenience. Then, we have the relation

$$
2 b_{1 j_{i}} b_{1 j_{i+1}}=h_{j_{i}} b_{1 j_{i}}^{2} .
$$

Suppose one of $b_{1 j_{i}}$ for $i=1, \ldots, k$ is zero. Then, from (4-4), all others must be zero, too. By a similar argument applied to the second relation $\phi^{-1}\left(f_{2}\right)=c_{12} \phi^{-1}\left(x_{1}\right)$, we can see that $b_{\ell j_{i}}=0$ for all $\ell=1, \ldots, n$ and $i=1, \ldots, k$. Thus, $\operatorname{det} B=0$, which is a contradiction. Therefore, all $b_{1 j_{i}}$ are nonzero for $i=1, \ldots, k$, and, hence, so are $h_{j_{i}}$ 's. Then, $-1=\Xi=1+(-1)^{k} \prod_{i=1}^{k} h_{j_{i}}$. Thus, $(-1)^{k} \prod_{i=1}^{k} h_{j_{i}}=-2$. By multiplying each side of Equation (4-4) for all $i=1, \ldots, k$, we have

$$
2^{k}\left(\prod_{i=1}^{k} b_{1 j_{i}}\right)^{2}=(-1)^{k+1} 2\left(\prod_{i=1}^{k} b_{1 j_{i}}\right)^{2}
$$

which is a contradiction. This proves the claim.

Therefore, the theorem follows from [12, Lemma 3.3].

It was shown by the authors and Masuda [5] that three-stage Bott manifolds are cohomologically rigid; that is, if $M$ and $N$ are two three-stage Bott manifolds whose cohomology rings are isomorphic, then they are diffeomorphic. The following corollary shows the cohomological rigidity of the class of 6-dimensional quasitoric manifolds whose cohomology rings are BQ-algebras over $\mathbb{Z}$. 
Theorem 4.3 Let $M$ and $N$ be 6-dimensional quasitoric manifolds whose cohomology rings are $B Q$-algebras over $\mathbb{Z}$. If $H^{*}(M) \cong H^{*}(N)$ as graded rings, then $M$ and $N$ are diffeomorphic.

Proof Since $H^{*}(M)$ and $H^{*}(N)$ are BQ-algebra over $\mathbb{Z}, M$ and $N$ are equivalent to 6-dimensional Bott manifolds. In particular, they are homeomorphic to 6-dimensional Bott manifolds. Since all quasitoric manifolds are simply connected, by the result of Wall [13] and Juppe [9], we can see that $M$ and $N$ are actually diffeomorphic to 6-dimensional Bott manifolds. Hence, the corollary follows from the above-mentioned result of [5].

\section{Cohomological rigidity of one-twist Bott manifolds}

In this section, we prove the cohomological rigidity of one-twist Bott manifolds. Let $\left\{B_{j} \mid 0 \leq j \leq n\right\}$ be a one-twist Bott tower. By Lemma 3.1, we may assume that $B_{n-1}=\left(\mathbb{C} P^{1}\right)^{n-1}$. Hence, $H^{*}\left(B_{n-1}\right) \cong \mathbb{Z}\left[x_{1}, \ldots, x_{n-1}\right] /\left\langle x_{j}^{2} \mid x=1, \ldots, n-1\right\rangle$. Let $M(\alpha)=B_{n}=P\left(\mathbb{C} \oplus \gamma^{\alpha}\right)$, where $\gamma^{\alpha}$ is the line bundle over $B_{n-1}$ with the first Chern class

$$
c_{1}\left(\gamma^{\alpha}\right)=\alpha=\sum_{i=1}^{n-1} a_{i} x_{i} \in H^{2}\left(B_{n-1}\right) .
$$

Theorem 5.1 Let $\alpha$ and $\beta$ be two elements of $H^{2}\left(B_{n-1}\right)$ where $B_{n-1}=\left(\mathbb{C} P^{1}\right)^{n-1}$, and let $M(\alpha)$ and $M(\beta)$ be one-twist Bott manifolds as defined above. Then, the following are equivalent.

(1) $M(\alpha)$ and $M(\beta)$ are diffeomorphic.

(2) $H^{*}(M(\alpha)) \cong H^{*}(M(\beta))$ as graded rings.

(3) There is an automorphism $\phi$ of $H^{*}\left(B_{n-1}\right)$ such that $\phi(\alpha) \equiv \beta \bmod 2$ and $\phi\left(\alpha^{2}\right)=\beta^{2}$.

(4) Let $\alpha=\sum_{i=1}^{n-1} a_{i} x_{i}$ and $\beta=\sum_{i=1}^{n-1} b_{i} x_{i}$. Then, there is a permutation $\sigma$ on $\{1, \ldots, n-1\}$ such that $a_{\sigma(i)} \equiv b_{i} \bmod 2$ for any $i$ and $\left|a_{\sigma(i)} a_{\sigma(j)}\right|=\left|b_{i} b_{j}\right|$ for any $i \neq j$.

Moreover, any isomorphism between $H^{*}(M(\alpha))$ and $H^{*}(M(\beta))$ preserves the total Pontrjagin classes of $M(\alpha)$ and $M(\beta)$.

As an easy corollary of Lemma 3.1 and Theorem 5.1, we have the following theorem. 
Theorem 5.2 If $M$ and $N$ are two one-twist Bott manifolds whose cohomology rings are isomorphic, then $M$ and $N$ are diffeomorphic.

Before we prove Theorem 5.1, let us note that

$$
H^{*}(M(\alpha))=\mathbb{Z}\left[x_{1}, \ldots, x_{n-1}, y_{\alpha}\right] /\left\langle x_{1}^{2}, \ldots, x_{n-1}^{2}, y_{\alpha}^{2}-\alpha y_{\alpha}\right\rangle,
$$

where $y_{\alpha}$ is the first Chern class of the tautological bundle of $P\left(\mathbb{C} \oplus \gamma^{a}\right)$. Moreover, its total Pontrjagin class is

$$
\begin{aligned}
P(M(\alpha)) & =\left(1+y_{\alpha}\right)^{2}\left(1+\left(y_{\alpha}-\alpha\right)^{2}\right) \\
& =1+\alpha^{2} .
\end{aligned}
$$

We first need the following lemma.

Lemma 5.3 The following are equivalent.

(1) $H^{*}(M(\alpha): \mathbb{Q}) \cong H^{*}\left(\left(\mathbb{C} P^{1}\right)^{n}: \mathbb{Q}\right)$.

(2) There is an element $u \in H^{*}\left(B_{n-1}: \mathbb{Q}\right)$ such that $\left(y_{\alpha}+u\right)^{2}=0$ in $H^{*}(M(\alpha): \mathbb{Q})$.

(3) $\alpha=a_{i} x_{i}$ for some $i=1, \ldots, n-1$.

There are two diffeomorphism types in this case, and $H^{*}(M(\alpha)) \cong H^{*}\left(\left(\mathbb{C} P^{1}\right)^{n}\right)$ if and only if $a_{i}$ is even in (3) above.

Proof $(1) \Rightarrow(2)$ Since there are $n$ linearly independent elements in the vector space $H^{2}\left(\left(\mathbb{C} P^{1}\right)^{n}: \mathbb{Q}\right)$ whose squares are zero, so are $H^{2}(M(\alpha): \mathbb{Q})$. From $(5-1)$, there are $n-1$ linearly independent elements $x_{1}, \ldots, x_{n-1}$ in $H^{2}(M(\alpha): \mathbb{Q})$ whose squares are zero. Thus, there is one more linearly independent element $w=\sum_{i=1}^{n-1} c_{i} x_{i}+c_{n} y_{\alpha} \in$ $H^{2}(M(\alpha): \mathbb{Q})$ such that $w^{2}=0$. Since $w$ is linearly independent from $x_{1}, \ldots, x_{n-1}$, the coefficient $c_{n}$ of $y_{\alpha}$ is non-zero. Let $u=c_{n}^{-1}\left(\sum_{i=1}^{n-1} c_{i} x_{i}\right) \in H^{2}\left(B_{n-1}: \mathbb{Q}\right)$. Then $\left(y_{\alpha}+u\right)^{2}=\left(c_{n}^{-1}\right)^{2} w^{2}=0$.

(2) $\Rightarrow$ (3) Let $u=\sum_{i=1}^{n-1} d_{i} x_{i}$ such that $\left(y_{\alpha}+u\right)^{2}=0$. Then,

$$
\begin{aligned}
0 & =\left(y_{\alpha}+\sum_{i=1}^{n-1} d_{i} x_{i}\right)^{2} \\
& =y_{\alpha}^{2}+2 \sum_{i<j}^{n-1} d_{i} d_{j} x_{i} x_{j}+2 \sum_{i=1}^{n-1} d_{i} x_{i} y_{a} \\
& =2 \sum_{i<j}^{n-1} d_{i} d_{j} x_{i} x_{j}+\left(2 \sum_{i=1}^{n-1} d_{i} x_{i}+\alpha\right) y_{\alpha} .
\end{aligned}
$$


This implies that $d_{i} d_{j}=0$ for all $i \neq j$, and $2 \sum_{i=1}^{n-1} d_{i} x_{i}+\alpha=0$. From the first condition, at most one, say $d_{i}$ is non-zero. From the second condition, we have $0=2 d_{i} x_{i}+\alpha$. If we set $a_{i}=-2 d_{i}$, then (3) follows.

(3) $\Rightarrow$ (1) If $\alpha=a_{i} x_{i}$, then $M(\alpha)$ is diffeomorphic to $B_{2} \times\left(\mathbb{C} P^{1}\right)^{n-2}$, where $B_{2}=$ $P\left(\mathbb{C} \oplus \gamma^{a_{i}}\right) \rightarrow \mathbb{C} P^{1}$. Here, $\gamma$ is the tautological line bundle over $\mathbb{C} P^{1}$. However, it is well-known that there are exactly two diffeomorphism type of $B_{2}$ depending on the parity of $a_{i}$. Namely, if $a_{i}$ is even, then $B_{2} \cong\left(\mathbb{C} P^{1}\right)^{2}$, and if $a_{i}$ is odd, then $B_{2}$ is diffeomorphic to a Hirzebruch surface $\mathcal{H}$. In the former case, $H^{*}(M(\alpha): \mathbb{Q})$ is trivially isomorphic to $H^{*}\left(\left(\mathbb{C} P^{1}\right)^{n}: \mathbb{Q}\right)$, and in the latter case

$$
\begin{aligned}
H^{*}(\mathcal{H}: \mathbb{Q}) & \cong \mathbb{Q}\left[x_{1}, x_{2}\right] /\left\langle x_{1}^{2}, x_{2}^{2}-x_{1} x_{2}\right\rangle \\
& \cong \mathbb{Q}\left[x_{1}, x_{2}\right] /\left\langle x_{1}^{2},\left(x_{2}-\frac{1}{2} x_{1}\right)^{2}\right\rangle \\
& \cong H^{*}\left(\left(C P^{1}\right)^{2}: \mathbb{Q}\right),
\end{aligned}
$$

which proves the lemma.

We now prove Theorem 5.1.

Proof of Theorem $5.1 \quad(2) \Rightarrow(3) \quad$ Let $\phi: H^{*}(M(\alpha)) \rightarrow H^{*}(M(\beta))$ be an isomorphism. In the case when $H^{*}(M(\alpha): \mathbb{Q}) \cong H^{*}\left(\left(\mathbb{C} P^{1}\right)^{n}: \mathbb{Q}\right)$, Lemma 5.3 shows that there are only two diffeomorphism types for $M(\alpha)$, which are $\left(\mathbb{C} P^{1}\right)^{n}$ and $\mathcal{H} \times\left(\mathbb{C} P^{1}\right)^{n-2}$, where $\mathcal{H}$ is the Hirzebruch surface. For these two types, we can see easily that $(2) \Rightarrow(3)$.

Therefore, we may assume that $H^{*}(M(\alpha): \mathbb{Q}) \cong H^{*}(M(\beta)$ : $\mathbb{Q})$ is not isomorphic to $H^{*}\left(\left(\mathbb{C} P^{1}\right)^{n}: \mathbb{Q}\right)$. For each $x_{i} \in H^{2}\left(B_{n-1}\right) \subset H^{2}(M(\alpha))$ as in (5-1), we have $\phi\left(x_{i}\right)^{2}=0$ in $H^{*}(M(\beta))$. On the other hand, since $x_{1}, \ldots, x_{n-1}, y_{\beta}$ are generators of $H^{*}(M(\beta))$, we can write $\phi\left(x_{i}\right)=b_{i 1} x_{1}+\cdots+b_{i n-1} x_{n-1}+b_{i n} y_{\beta}$. Then, by Lemma 5.3, the coefficient $b_{i n}$ of $y_{\beta}$ must vanish for $i=1, \ldots, n-1$. This means that any isomorphism $\phi: H^{*}(M(\alpha)) \rightarrow H^{*}(M(\beta))$ must preserve the subring $H^{*}\left(B_{n-1}\right)$. Therefore, $\phi\left(y_{\alpha}\right)= \pm y_{\beta}+w$ for some $w \in H^{2}\left(B_{n-1}\right)$. If necessary, by composing $\phi$ with an automorphism of $H^{*}(M(\beta))$ fixing $H^{*}\left(B_{n-1}\right)$ and sending $y_{\beta}$ to $-y_{\beta}$, we may assume that $\phi\left(y_{\alpha}\right)=y_{\beta}+w$. It follows that

$$
\phi\left(y_{\alpha}^{2}\right)=\left(y_{\beta}+w\right)^{2}=y_{\beta}^{2}+2 w y_{\beta}+w^{2}=(\beta+2 w) y_{\beta}+w^{2} .
$$

On the other hand, we have

$$
\phi\left(y_{\alpha}^{2}\right)=\phi\left(\alpha y_{\alpha}\right)=\phi(\alpha)\left(y_{\beta}+w\right) .
$$


Comparing (5-3) and (5-4), we obtain

$$
\phi(\alpha)=\beta+2 w \quad \text { and } \quad w^{2}=\phi(\alpha) w .
$$

The first equation of (5-5) implies $\phi(\alpha) \equiv \beta \bmod 2$. By plugging the first equation into the second of (5-5), we can see that $\beta w=-w^{2}$. Hence, $\phi\left(\alpha^{2}\right)=(\beta+2 w)^{2}=$ $\beta^{2}+4 \beta w+4 w^{2}=\beta^{2}$. Hence, (2) $\Rightarrow(3)$ is proved.

(3) $\Rightarrow$ (2) Suppose there is an automorphism $\phi$ on $H^{*}\left(B_{n-1}\right)$ such that $\phi(\alpha) \equiv \beta$ $\bmod 2$ and $\phi\left(\alpha^{2}\right)=\beta^{2}$. Let $\phi(\alpha)=\beta+2 w$ for some $w \in H^{2}\left(B_{n-1}\right)$. If we define $\phi\left(y_{\alpha}\right)=y_{\beta}+w$, then we can see easily that $\phi$ defines an isomorphism from $H^{*}(M(\alpha))$ to $H^{*}(M(\beta))$. This proves $(3) \Rightarrow(2)$.

$(1) \Rightarrow(2)$ This implication is obvious.

(2) $\Rightarrow(1)$ Suppose $H^{*}(M(\alpha))$ is isomorphic to $H^{*}(M(\beta))$. By (2) $\Rightarrow(3)$, there is an automorphism $\phi$ on $H^{*}\left(B_{n-1}\right) \cong \mathbb{Z}\left[x_{1}, \ldots, x_{n-1} /\left\langle x_{j}^{2} \mid j=1, \ldots, n-1\right\rangle\right.$. But it is easy to see that any automorphism on $\mathbb{Z}\left[x_{1}, \ldots, x_{n-1} /\left\langle x_{j}^{2} \mid j=1, \ldots, n-1\right\rangle\right.$ is generated by a permutation on the generators $x_{1}, \ldots, x_{n-1}$, possibly changing their signs. Such automorphism on the ring $H^{*}\left(B_{n-1}\right)$ is clearly induced by a selfdiffeomorphism $f$ on $B_{n-1}=\left(\mathbb{C} P^{1}\right)^{n-1}$; that is, $f^{*}=\phi$. The diffeomorphism $f$ induces a fiber bundle isomorphism between $\gamma^{\alpha}$ and $f^{*}\left(\gamma^{\alpha}\right)$; hence, it induces a diffeomorphism between $M(\alpha)$ and $M(\phi(\alpha))$. Therefore, for simplicity, we may assume the automorphism $\phi$ on $H^{*}\left(B_{n-1}\right)$ is the identity, such that $\alpha \equiv \beta \bmod 2$ and $\alpha^{2}=\beta^{2}$.

Since $\alpha \equiv \beta \bmod 2$, there is an element $w \in H^{2}\left(B_{n-1}\right)$ such that $2 w=\alpha-\beta$. Now, let $\xi_{1}=\gamma^{\alpha} \oplus \mathbb{C}$ and $\xi_{2}=\gamma^{w}\left(\gamma^{\beta} \oplus \mathbb{C}\right)$. Then, their first Chern classes are equal because $c_{1}\left(\xi_{1}\right)=\alpha=\beta+2 w=c_{1}\left(\xi_{2}\right)$. Their second Chern classes are $c_{2}\left(\xi_{1}\right)=0$ and $c_{2}\left(\xi_{2}\right)=$ $w(\beta+w)=0$, which follows from (5-5). Therefore, $\xi_{1} \cong \xi_{2}$ by Proposition 2.5, and, hence, $M(\alpha)=P\left(\xi_{1}\right) \cong P\left(\xi_{2}\right)=P\left(\gamma^{w}\left(\gamma^{\beta} \oplus \mathbb{C}\right)\right) \cong P\left(\gamma^{\beta} \oplus \mathbb{C}\right)=M(\beta)$. This proves $(2) \Rightarrow(1)$.

That $(3) \Leftrightarrow(4)$ is obvious.

If $\phi: H^{*}(M(\alpha)) \rightarrow H^{*}(M(\beta))$ is any isomorphism, the proof (2) $\Rightarrow(3)$ shows that $\phi\left(\alpha^{2}\right)=\beta^{2}$. Hence, by the identity (5-2), the isomorphism $\phi$ preserves the Pontrjagin classes of $M(\alpha)$ and $M(\beta)$.

By putting all the results together, we can conclude the following cohomological rigidity result for quasitoric manifolds.

Theorem 5.4 Let $M$ and $N$ be $2 n$-dimensional quasitoric manifolds whose cohomology algebras are $B Q$-algebra of rank $n$ over $\mathbb{Z}$ with cohomological complexities equal to 1 . If $H^{*}(M) \cong H^{*}(N)$, then $M$ and $N$ are homeomorphic. 
Proof By Theorem 3.2 and Corollary 3.3 both $M$ and $N$ are equivalent to onetwist $n$-stage Bott manifolds. By Theorem 5.1, those one-twist Bott manifolds are diffeomorphic. Hence, $M$ and $N$ are homeomorphic.

\section{BQ-algebra over $\mathbb{Z}_{(2)}$}

All the results in previous sections are concerned with BQ-algebras over $\mathbb{Z}$. In this section, we remark that these results are still true for BQ-algebras over the localized $\operatorname{ring} \mathbb{Z}_{(2)}$ at 2 .

A BQ-algebra over $\mathbb{Z}$ is defined in Definition 4.1. However, this definition can be extended to any commutative ring $R$. Namely, a BQ-algebra $S$ of rank $n$ over $R$ is a graded $R$-algebra with generators $x_{1}, \ldots, x_{n}$ of degree 2 such that

(1) $x_{k}^{2}=\sum_{i<k} c_{i k} x_{i} x_{k}$, where $c_{i k} \in R$ for $1 \leq k \leq n$, (in particular $x_{1}^{2}=0$,) and (2) $\prod_{i=1}^{n} x_{i} \neq 0$.

The $R$-complexity of $S$ is the number of $k$ 's such that $x_{k}^{2} \neq 0$ in the above condition (1) for all possible choices of generator sets $\left\{x_{1}, \ldots, x_{n}\right\}$. Note that the cohomology ring $H^{*}(M, R)$ of a quasitoric manifold $M$ is a BQ-algebra over $R$. If $R=\mathbb{Z}$ and $M$ is a Bott manifold, the cohomological complexity of $M$ defined in Section 3 is the $\mathbb{Z}$-complexity of $H^{*}(M: \mathbb{Z})$.

In Theorem 3.2, we show that the twist number of a Bott manifold $M$ is equal to the cohomological complexity of $M$. If we examine the proof carefully, the proof is based on arguments on whether the coefficients are even or odd. Therefore we can see easily that the same argument works if the integer coefficients are replaced by the localized ring $\mathbb{Z}_{(2)}$ at 2. Therefore, Theorem 3.2 and Corollary 3.3 can be extended as follows.

Theorem 6.1 Let $M$ be a Bott manifold. Then, the twist number of $M$ is well-defined and is equal to the $\mathbb{Z}_{(2)}$-complexity of the $B Q$-algebra $H^{*}\left(M: \mathbb{Z}_{(2)}\right)$. In particular, the $\mathbb{Z}$-complexity of $H^{*}(M: \mathbb{Z})$ is equal to the $\mathbb{Z}_{(2)}$-complexity of $H^{*}\left(M: \mathbb{Z}_{(2)}\right)$.

In Theorem 4.2, it is shown that if $M$ is a quasitoric manifold whose integral cohomology ring is a BQ-algebra over $\mathbb{Z}$, then $M$ is equivalent to a Bott manifold. In its proof, the only place where the property of integral coefficients differs from that of rational coefficients is used is where $a_{s t} a_{t s}=2$ implies $a_{s t}= \pm 1$ and $a_{t s}= \pm 2$ right after Equation (4-2). However, this is still true if the coefficient ring is $\mathbb{Z}_{(2)}$, the integer ring localized at 2 . Therefore, Theorem 4.2 is still true if the coefficient ring is $\mathbb{Z}_{(2)}$. Therefore, Theorem 4.2 can be extended as follows. 
Theorem 6.2 Let $M$ be a $2 n$-dimensional quasitoric manifold over $P$, and let $A$ be the characteristic matrix of $M$. Then, the following are equivalent.

(1) $M$ is equivalent to an $n$-stage Bott manifold.

(2) $H^{*}(M: \mathbb{Z})$ is a $B Q$-algebra of rank $n$ over $\mathbb{Z}$.

(3) $H^{*}\left(M: \mathbb{Z}_{(2)}\right)$ is a $B Q$-algebra of rank $n$ over $\mathbb{Z}_{(2)}$.

(4) $P$ is combinatorially equivalent to the cube $I^{n}$, and $A$ is an $n \times n$ matrix conjugate to an upper triangular matrix by a permutation matrix.

If we examine the proof of Theorem 5.1 carefully, we can also see that a similar proof works for the following claim: if $M(\alpha)$ and $M(\beta)$ are one-twist Bott manifolds with $H^{*}\left(M(\alpha): \mathbb{Z}_{(2)}\right) \cong H^{*}\left(M(\beta): \mathbb{Z}_{(2)}\right)$, then they are diffeomorphic. So combining this claim together with Theorems 6.1 and 6.2 , we can obtain the following theorem.

Theorem 6.3 Let $M$ and $N$ be $2 n$-dimensional quasitoric manifolds whose cohomology rings are $B Q$-algebras of rank $n$ over $\mathbb{Z}_{(2)}$ with $\mathbb{Z}_{(2)}$-complexities less than or equal to 1 . If $H^{*}\left(M: \mathbb{Z}_{(2)}\right) \cong H^{*}\left(N: \mathbb{Z}_{(2)}\right)$, then $M$ and $N$ are homeomorphic.

In the proof of the cohomological rigidity of three-stage Bott manifolds in [5], Wall and Juppe's results on classification of simply connected 6-dimensional manifolds is essential. However, recently, a different but direct proof of the cohomological rigidity of three-stage Bott manifolds has been found, and a similar proof also works for the claim that two three-stage Bott manifolds with isomorphic $\mathbb{Z}_{(2)}$-cohomology rings are diffeomorphic. Therefore, using the same argument as above, we have the following theorem.

Theorem 6.4 Let $M$ and $N$ be 6-dimensional quasitoric manifolds whose $\mathbb{Z}_{(2)}$ cohomology rings are $B Q$-algebras over $\mathbb{Z}_{(2)}$. If $H^{*}\left(M: \mathbb{Z}_{(2)}\right) \cong H^{*}\left(N: \mathbb{Z}_{(2)}\right)$ as graded rings, then $M$ and $N$ are diffeomorphic.

More precise argument for Theorem 6.4 will be shown elsewhere.

\section{Acknowledgement}

The first author was supported by the Japanese Society for the Promotion of Sciences (JSPS grant P09023), and the second author was partially supported by the Basic Science Research Program through the National Research Foundation of Korea (NRF) funded by the Ministry of Education, Science, and Technology (2010-0001651). 


\section{References}

[1] R Bott, H Samelson, Applications of the theory of Morse to symmetric spaces, Amer. J. Math. 80 (1958) 964-1029 MR0105694

[2] V M Buchstaber, T E Panov, Torus actions and their applications in topology and combinatorics, University Lecture Series 24, American Mathematical Society, Providence, RI (2002) MR1897064

[3] S Choi, M Masuda, S Oum, Classification of real Bott manifolds and acyclic digraphs arXiv: 1006.4658

[4] S Choi, M Masuda, D Y Suh, Quasitoric manifolds over a product of simplices, Osaka J. Math. 47 (2010) 109-129 MR2666127

[5] S Choi, M Masuda, D Y Suh, Topological classification of generalized Bott towers, Trans. Amer. Math. Soc. 362 (2010) 1097-1112 MR2551516

[6] S Choi, T Panov, D Y Suh, Toric cohomological rigidity of simple convex polytopes, J. Lond. Math. Soc. (2) 82 (2010) 343-360 MR2725043

[7] M W Davis, T Januszkiewicz, Convex polytopes, Coxeter orbifolds and torus actions, Duke Math. J. 62 (1991) 417-451 MR1104531

[8] M Grossberg, Y Karshon, Bott towers, complete integrability, and the extended character of representations, Duke Math. J. 76 (1994) 23-58 MR1301185

[9] P E Jupp, Classification of certain 6-manifolds, Proc. Cambridge Philos. Soc. 73 (1973) 293-300 MR0314074

[10] Y Kamishima, M Masuda, Cohomological rigidity of real Bott manifolds, Algebr. Geom. Topol. 9 (2009) 2479-2502 MR2576506

[11] M Masuda, Cohomological non-rigidity of generalized real Bott manifolds of height 2, Tr. Mat. Inst. Steklova 268 (2010) 252-257 MR2724345

[12] M Masuda, T E Panov, Semi-free circle actions, Bott towers, and quasitoric manifolds, Mat. Sb. 199 (2008) 95-122 MR2452268

[13] C T C Wall, Classification problems in differential topology V: On certain 6-manifolds, Invent. Math. 1 (1966) 355-374 MR0215313 Correction: Invent. Math. 2 (1966) 306

Department of Mathematics, Ajou University,

San 5, Woncheon-dong, Yeongtong-gu, Suwon 443-749, Republic of Korea

Department of Mathematical Sciences, KAIST

335 Gwahangno, Yu-sung Gu, Daejeon 305-701, Republic of Korea

schoi@ajou.ac.kr, dysuh@math.kaist.ac.kr

Received: 24 April $2010 \quad$ Revised: 31 October 2010 